\title{
"MIRAI-21" ANALYTICAL ELECTRON MICROSCOPE - PERFORMANCE OF THE MONOCHROMATOR -
}

\author{
M. Mukai, T. Kaneyama, T. Tomita, K. Tsuno, M. Terauchi ${ }^{*}$, K. Tsuda ${ }^{*}$, K. Saitoh ${ }^{*}$, M. Naruse, T. \\ Honda and M. Tanaka*
}

JEOL Ltd., 3-1-2 Musashino, Akishima, Tokyo 196-8558, Japan

*Institute of Multidisciplinary Research for Advanced Materials, Tohoku University, 2-1-1 Katahira, Aoba-ku, Sendai 980-8577, Japan

We have been developing a high-energy-resolution analytical electron microscope under the project "MIRAI-21", which enables us to investigate both crystal- and electronic-structures of advanced materials in nanometer scale areas ${ }^{1,2,3}$. MIRAI means "Future" in Japanese and the abbreviation of Microscope for Innovative Research and Advanced Investigation. This microscope is constructed based on the JEM-2010FEF, and equipped with a newly developed Wien-filter monochromator and an improved $\Omega$-filter analyzer. The microscope was aimed for a point resolution of $0.19 \mathrm{~nm}$ and an energy resolution of $0.2 \mathrm{eV}$ at less than a $2 \mathrm{~nm}$ diameter probe. In this paper, the basic design and the test results of the monochromator are reported.

A new monochromator is located between the extraction anode of the $\mathrm{ZrO} / \mathrm{W}$ emitter and the acceleration tube. Figure 1 shows the arrangement of electric plates (+ and -) and magnetic poles ( $\mathrm{S}$ and $\mathrm{N}$ ) of the octopole-type Wien filter. The monochromator consists of two octopole-type Wien-filters ${ }^{4}$ and a slit for energy selection, which is placed between the two filters. The upper Wien-filter disperses the incident electron beam and forms a line focused image on the energy selection slit. The lower Wien-filter cancels out the energy dispersion of the upper filter and forms a round shaped electron beam at the exit of the monochromator. It should be noted that each filter can generate a quadrupole field to control the beam shape.

Figure 2 shows the zero-loss peak profiles for different energy-selection settings. A peak width of $0.71 \mathrm{eV}$ was obtained without energy selection. The other two were obtained different widths of the energy selection slit. The best energy resolution is $0.19 \mathrm{eV}$ at present. The beam current of the profile for a $0.19 \mathrm{eV}$ energy width is about $1 / 10$ of that of the profile of $0.71 \mathrm{eV}$.

Figure 3 shows beam shapes (lower) and their intensity profiles (upper) at the specimen position. The beam shapes were obtained by applying a quadrupole E-field in the second Wien-filter. The beam shape of Fig.3(a) was obtained without energy selection. The beam does not show a circular shape, which is due to imperfect setting of the quadrupole correction field. Figure 3(b) shows a circular beam shape of less than $1 \mathrm{~nm}$ in diameter obtained at an energy selection setting of $0.26 \mathrm{eV}$. Detailed results will be reported at the meeting.

The present study is supported by the project "Technology Transfer D98-04" of Japan Science and Technology Corporation.

\section{References}

1. M. Tanaka et.al.: Inst. Phys. Conf. Ser., $\underline{165}, 217-218$ (2000).

2. M. Mukai et al.: Proc. ICEM-15, vol.3, 321-322 (2002).

3. M. Tanaka et al.: Microsc. Microanal., $\underline{8}$ (Suppl. 2), 68-69 (2002).

4. M. Terauchi, M. Tanaka, K. Tsuno and M. Ishida: J. Microscopy, 194, 203-209 (1999). 


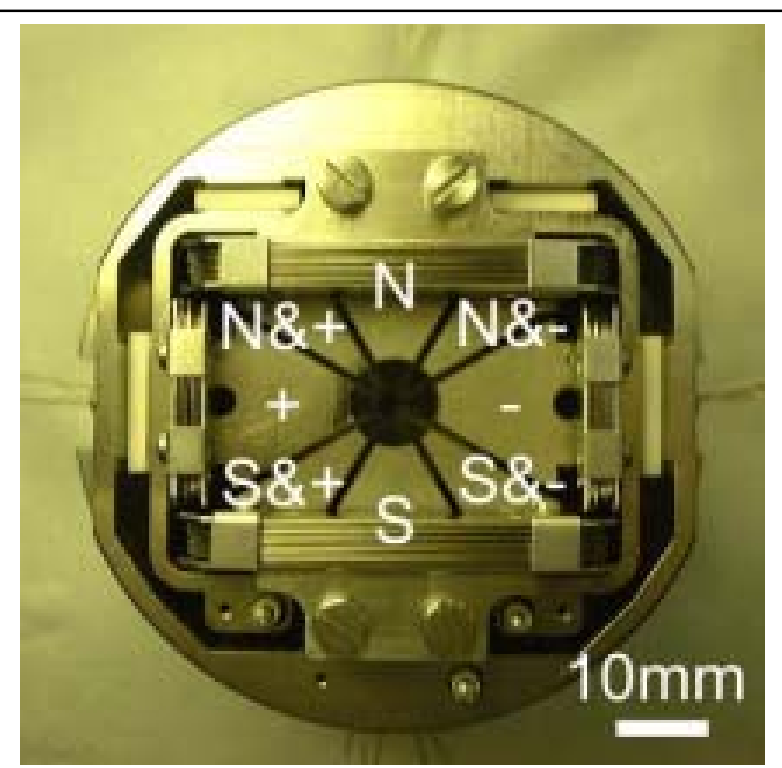

Figure 1. Octopole-type Wien filter.

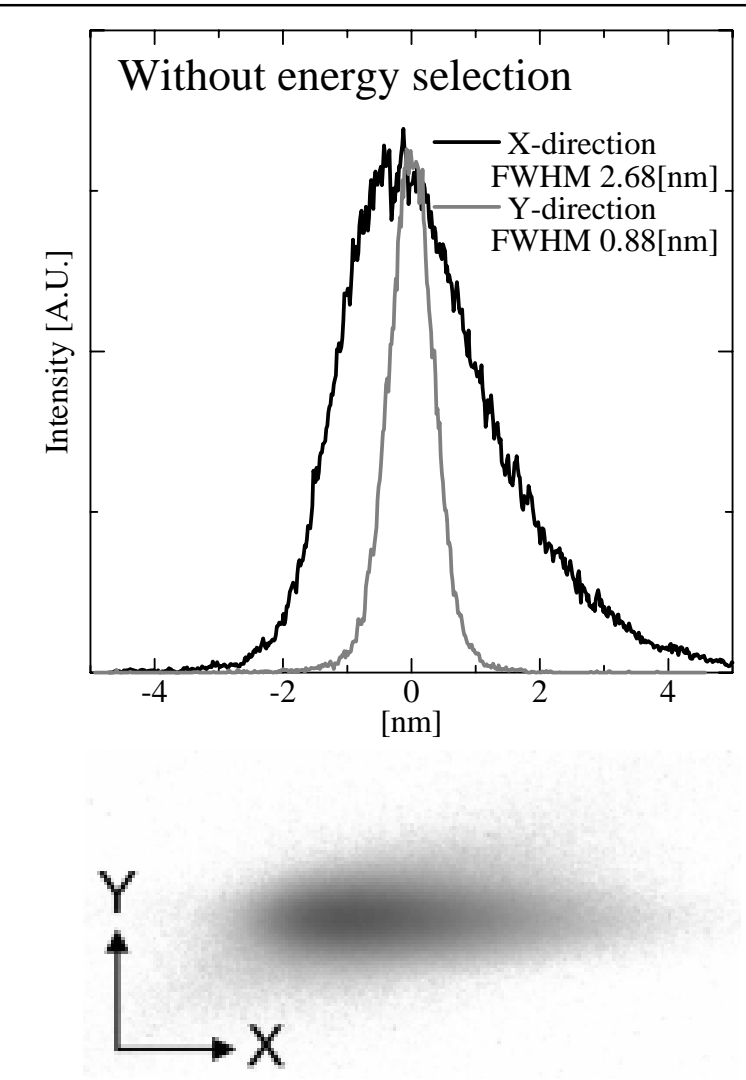

(a)
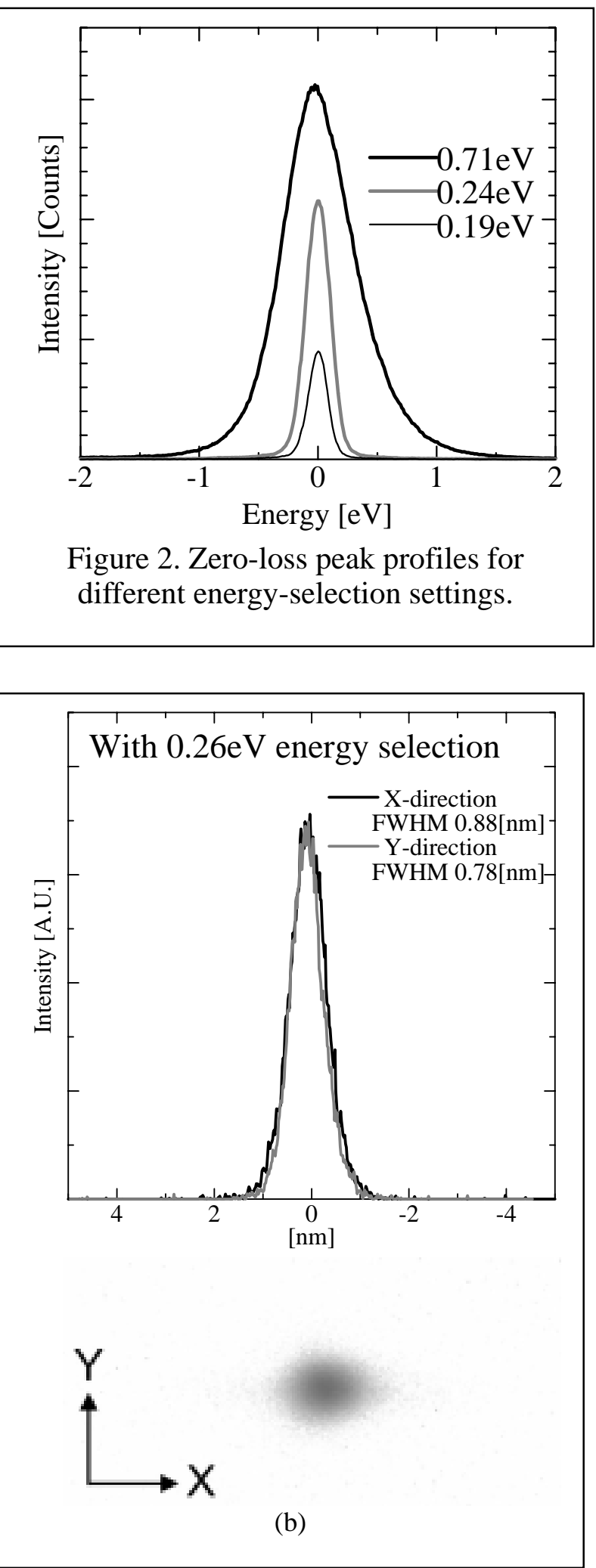

Figure 3. Beam shapes at the specimen position (lower) and its intensity profiles (upper). (a) was obtained without energy selection. (b) a circular beam has a width of less than $1 \mathrm{~nm}$. The beam was obtained with an energy selection setting of $0.26 \mathrm{eV}$. 